\title{
Severe hypoglycemia and finger clubbing in a patient with a BRCA1 mutation in a solitary fibrous tumor: a case report
}

\author{
Wei Guo ${ }^{1}$, Ying $\mathrm{Ji}^{1}$, Lei Guo ${ }^{2}$, Shunan Che ${ }^{3}$, Qilin Huai ${ }^{4}$, Kun Yang ${ }^{1}$, Fengwei Tan ${ }^{1}$, Qi Xue ${ }^{1}$, Shugeng Gao ${ }^{1}$, \\ Jie $\mathrm{He}^{1}$ \\ ${ }^{1}$ Department of Thoracic Surgery, National Cancer Center/National Clinical Research Center for Cancer/Cancer Hospital, Chinese Academy of \\ Medical Sciences and Peking Union Medical College, Beijing, China; ${ }^{2}$ Department of Pathology, National Cancer Center/National Clinical Research \\ Center for Cancer/Cancer Hospital, Chinese Academy of Medical Sciences and Peking Union Medical College, Beijing, China; ${ }^{3}$ Department of \\ Radiology, National Cancer Center/National Clinical Research Center for Cancer/Cancer Hospital, Chinese Academy of Medical Sciences and \\ Peking Union Medical College, Beijing, China; ${ }^{4}$ Department of Graduate School, Zunyi Medical University, Zunyi, China \\ Correspondence to: Jie He; Shugeng Gao; Qi Xue. Department of Thoracic Surgery, National Cancer Center/National Clinical Research Center for \\ Cancer/Cancer Hospital, Chinese Academy of Medical Sciences and Peking Union Medical College, Panjiayuannanli No. 17, Chaoyang District, \\ Beijing 100021, China. Email: prof.jiehe@gmail.com; gaoshugeng@vip.sina.com; xueqi@cicams.ac.cn.
}

\begin{abstract}
Solitary fibrous tumors (SFTs) are rare tumors that stem from mesenchymal cells of submesothelial tissues belonging to the pleura. They can occur in many places such as the spinal canal, intracranial, neck, kidney, liver, pelvis, limbs and other places, most commonly in the chest and abdomen. Pleural SFTs are one of the most common types, and are common in middle-aged people. Pleural SFTs can have an insidious expression, such that the illness can progress for years before diagnosis. SFTs can induce paraneoplastic syndromes, such as reactive hypoglycemia [Doege-Potter syndrome (DPS)] or hypertrophic osteoarthropathy [Pierre-Marie-Bamberger syndrome (PMBS)]. In this article, we report a case study of a 51-year-old man with pleural SFTs. Preoperative imaging examinations, including chest X-ray, computed tomography (CT), and magnetic resonance imaging (MRI), showed a huge mass in the right thoracic cavity, compressing surrounding tissues and organs and may invade other tissues. In addition, he suffers from severe hypoglycemia and finger clubbing, and has successfully undergone a complete resection, and now attends regular follow-up appointments. The paraneoplastic syndromes have resolved, and no recurrence has been found. Importantly, we used next-generation sequencing (NGS) to explore the molecular characteristics of the patient's pathological tissue at the DNA level and mRNA level, and found that breast cancer gene 1 (BRAC1) mutations may be an important pathogenic factor.
\end{abstract}

Keywords: Solitary fibrous tumor (SFT); Doege-Potter syndrome (DPS); Pierre-Marie-Bamberger syndrome (PMBS); NAB2-STAT6 fusion; case report

Submitted Feb 25, 2021. Accepted for publication May 31, 2021.

doi: $10.21037 / \mathrm{atm}-21-914$

View this article at: https://dx.doi.org/10.21037/atm-21-914

\section{Introduction}

Solitary fibrous tumors (SFTs) are rare tumors that originate in mesenchymal tissues, and account for only $2 \%$ of all soft tissue tumors $(1,2)$. Despite widespread reports of SFTs in various parts of the body, they typically occur in the pleura (3). Clinically, most pleural SFTs have a slow growth rate. Due to a lack of obvious symptoms, they are often only detected accidently on chest radiographs. However, in some instances, pleural SFTs may manifest with compressive symptoms or, exceptionally, with paraneoplastic syndromes, such as reactive hypoglycemia [Doege-Potter syndrome (DPS)] or hypertrophic osteoarthropathy [Pierre-MarieBamberger syndrome (PMBS)] (3-5). The surgical resection of pleural SFTs is the most effective treatment for primary tumors and prevents the recurrence of DPS and PMBS $(6,7)$.

Here, we describe an occurrence of DPS and PMBS 
Table 1 Summary of the laboratory tests results of the case

\begin{tabular}{lccc}
\hline Serum concentration & Pre-operation value & Post-operation value & Reference range \\
\hline Glucose, $\mathrm{mmol} / \mathrm{L}$ & 2.04 & 4.41 & $3.61-5.11$ \\
Potasium, $\mathrm{mmol} / \mathrm{L}$ & 2.67 & 4.01 & $3.5-5.5$ \\
Insulin, ulU/mL & $<0.2$ & - & $2.6-24.9$ \\
C-peptide, $\mathrm{ng} / \mathrm{mL}$ & 0.07 & - & $0.78-1.89$ \\
GH, $\mathrm{ng} / \mathrm{mL}$ & 0.15 & - & $0.03-2.47$ \\
Direct $\mathrm{renin}, \mathrm{mU} / \mathrm{L}$ & 9.5 & - & $2.8-39.9$ \\
Aldosterone, $\mathrm{ng} / \mathrm{dL}$ & 1.9 & - & $3.0-23.6$ \\
Cortisol, $\mu \mathrm{g} / \mathrm{dL}$ & 11.16 & - & $4.4-19.9$ \\
ACTH, $\mathrm{pg} / \mathrm{mL}$ & 37.98 & - & $7.2-63.3$ \\
IGF I, $\mathrm{ng} / \mathrm{mL}$ & 108 & 347.82 & $87-283$ \\
IGF II, $\mu \mathrm{dL}$ & $1,228.71$ & - \\
\hline
\end{tabular}

C-peptide, GH, direct renin, aldosterone, cortisol, ACTH, and IGFI were not measured after operation. GH, growth hormone; ACTH, adrenocorticotropic hormone; IGF I, insulin-like growth factor I; IGF II, insulin-like growth factor II.

with a huge pleural SFT in an adult Chinese man. After radical resection of the causative tumor, the paraneoplastic symptoms disappeared quickly. In comparison with other literature reports, this case featured a huge tumor with DPS and PMBS. Additionally, the breast cancer gene 1 (BRAC1) mutation was detected in this case using next-generation sequencing (NGS). We present the following case in accordance with the CARE reporting checklist (available at https://dx.doi.org/10.21037/atm-21-914).

\section{Case presentation}

A healthy 51-year-old male presented to our hospital with a recurrent 2-month episode and symptoms history of severe hypoglycemia, including diaphoresis, tremor, anxiety, and loss of consciousness. A chest X-ray and computed tomography (CT) scan of his thorax at another hospital revealed a pleural mass in the patient's right thoracic cavity. There were no pulmonary complaints. No dramatic findings were observed on the physical examination other than finger clubbing and diminished breath sounds in the right middle and lower lobes of the lung. Laboratory tests, including peripheral blood examinations and tumor biomarkers, were within normal range, except that the level of blood potassium was $2.67 \mathrm{mmol} / \mathrm{L}$. Despite marked hypoglycemia $(1.4 \mathrm{mmol} / \mathrm{L})$, the serum insulin level $\mathrm{did}$ not reach $0.2 \mathrm{mIU} / \mathrm{mL}$ (normal range, $2.6-24.9 \mathrm{mIU} / \mathrm{mL}$ ), the $\mathrm{C}$-peptide level reached $0.07 \mathrm{ng} / \mathrm{mL}$ (normal range,
$0.78-1.89 \mathrm{ng} / \mathrm{mL}$ ), the serum insulin-like growth factor-II (IGF-II) level reached $1,228.71 \mathrm{mg} / \mathrm{dL}$, and the IGF-I level reached $108 \mathrm{ng} / \mathrm{mL}$ (see Table 1).

Repeated chest X-rays showed a huge mass in the right thoracic cavity (see Figure 1A,B). The thoracic CT scan revealed a mass with an unclear boundary that was compressing the right atrium, right pulmonary artery, and right superior pulmonary vein. The great heart vessel was also deviated to the left. Additionally, the right lung's middle lobe and lower lobe was compressed and inflated, and the local lung field near the mass was consolidated (see Figure 1C,D). The mass presented with high-degree intensity in $\mathrm{T} 2$ weighted image magnetic resonance imaging (MRI), and showed a heterogeneous signal increase on the dynamic MRI; however, there was no obvious enhancement in the necrosis area (see Figure 1E,F). Bone emission CT scans and MRI of the brain were unremarkable. From the preoperative image findings, we suspected that the tumor might have invaded other organs, including the lung, right atrium, right pulmonary artery, right superior pulmonary vein, and great heart vessel. A CT scan-guided transthoracic puncture biopsy was performed on the patient, and a microscopic examination of the specimen revealed spindle tumor cells. The tumor cells were slightly atypical, and the soft tissue tumors had lowgrade malignancy.

Next a, right posterolateral open thoracotomy was performed. We chose the left lateral position, and made a right posterolateral sixth intercostal incision into the chest. 

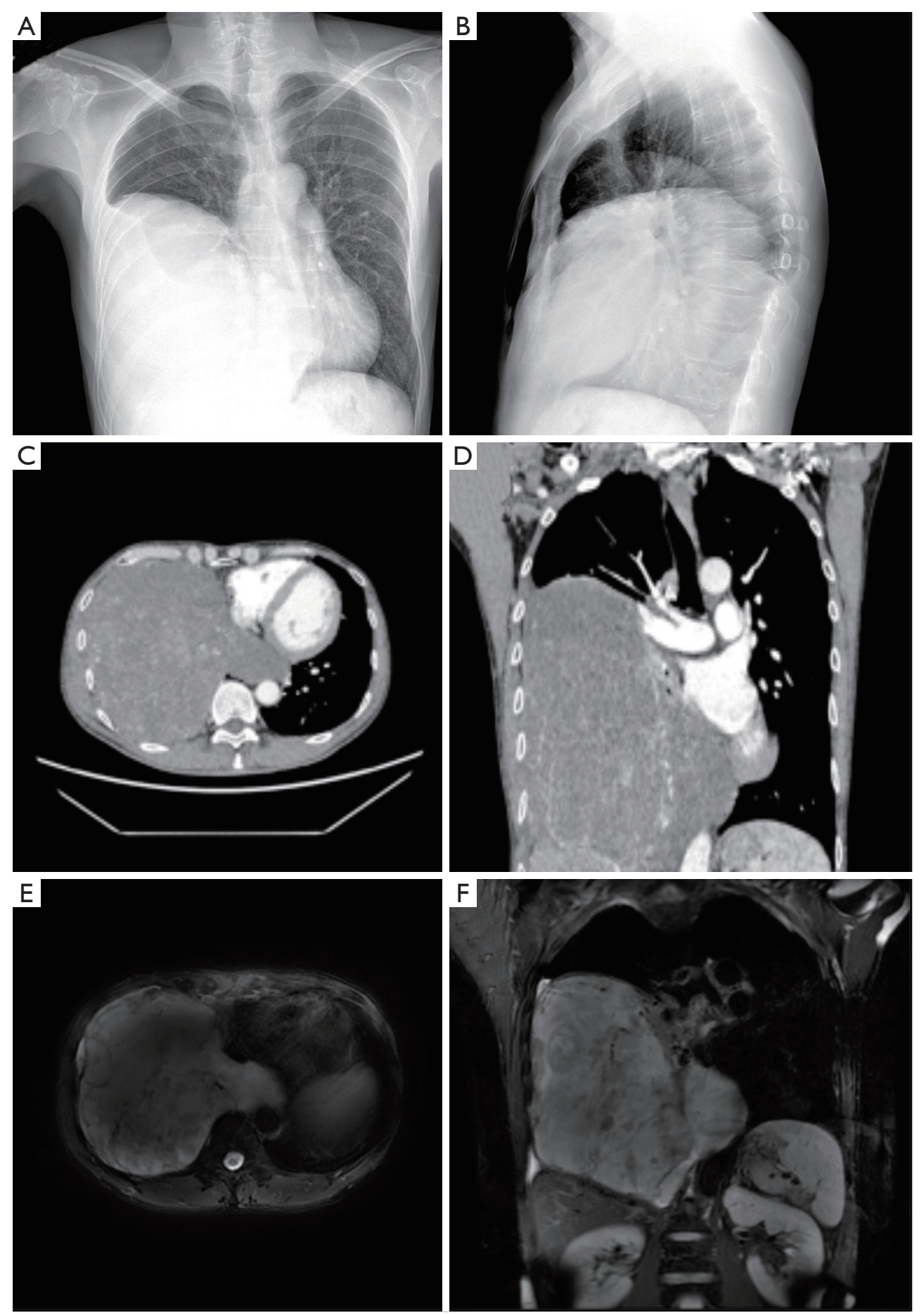

Figure 1 Imaging examination of patients with pleural solitary fibrous tumor (SFT). (A,B) A chest X-ray showed a huge mass in the right thoracic cavity; (C,D) thoracic computed tomography (CT) scan of the SFT; (E,F) magnetic resonance imaging (MRI) of the SFT. 


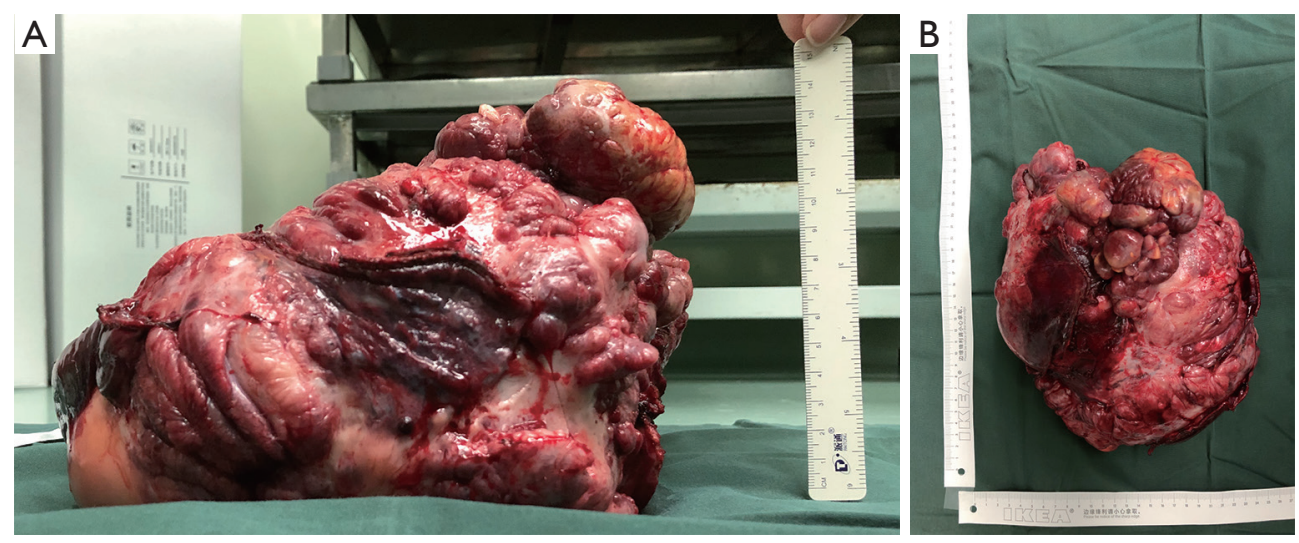

Figure 2 Macroscopic findings of resected tumor: the tumor measured approximately $22 \times 22 \times 11 \mathrm{~cm}^{3}$.
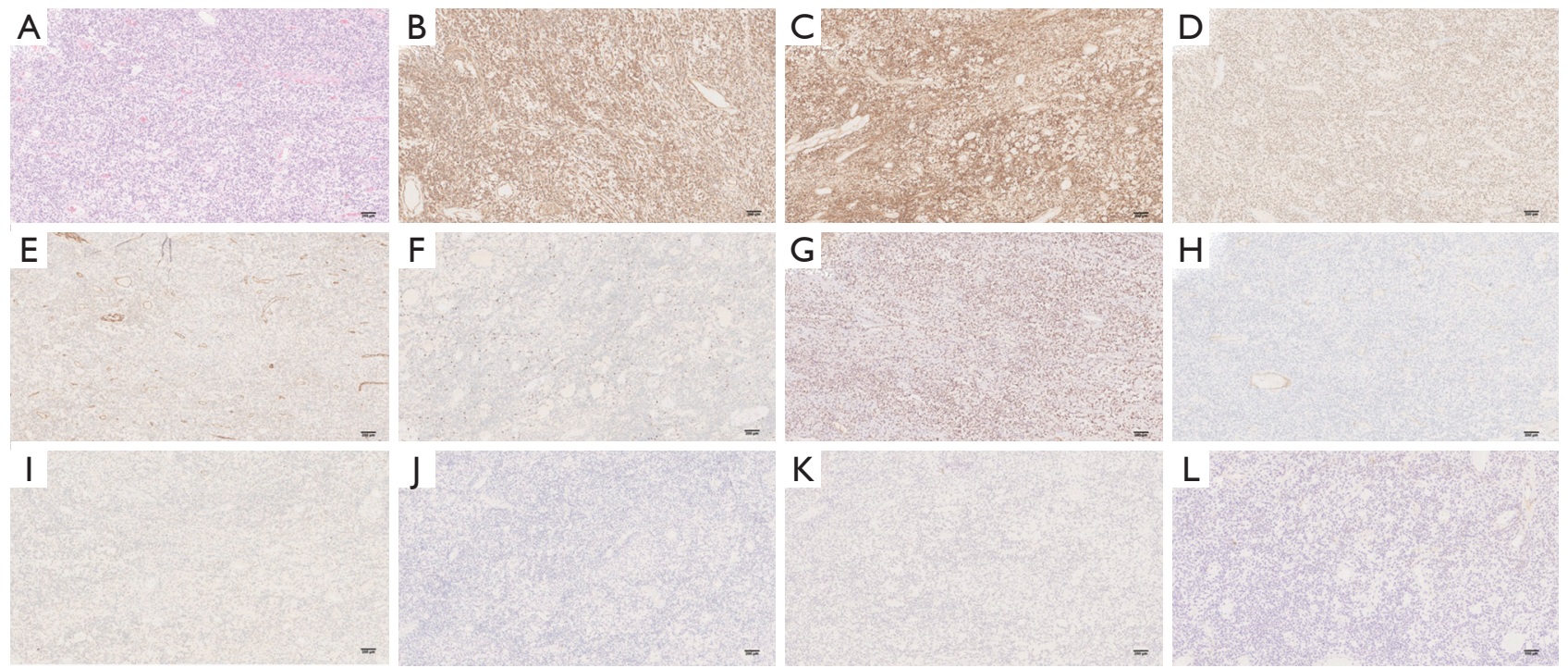

$\mathrm{H}$
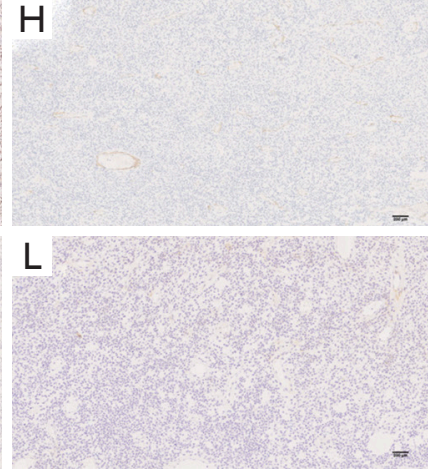

Figure 3 Histopathological findings showing spindle cells arranged in an organization lacking an obvious pattern. Hematoxylin-eosin (HE) staining (A) and immunohistochemistry of vimentin (B), bcl-2 (C), STAT6 (D), CD34 (E), Ki-67 (15\%) (F), IGF-2 (G), SMA (H), desmin (I), S-100 (J), calretinin (K) and AE1/AE3 (L).

The length of the wound was approximately $30 \mathrm{~cm}$. There were a small number of adhesions in the thoracic cavity. The tumor, which stemmed out of the anterior mediastinum, was in the accompanying right lung and diagram, and there was phrenic nerve invasion. A percentage of the lower lobe of the right lung, a proportion of the diagram, and the phrenic nerve were resected. The tumor, which measured $22 \times 22 \times 11 \mathrm{~cm}^{3}$ in size and weighed $3,500 \mathrm{~g}$, was encapsulated (see Figure 2). Histologic assessment of a lung biopsy specimen revealed a tumor composed of simple spindle-shaped cells with no obvious nuclear pleomorphism (less than 4 mitotic figures per 10 high-power fields). Necrosis was not observed. The immunohistochemical findings were as follows: vimentin $(+)$, Bcl-2 (+), STAT6 (+), CD34 (+), IGF-II (+), SMA (-), desmin (-), S-100 (-), calretinin (-), AE1/AE3 (-), and Ki67 labeling index 15\% (see Figure 3). A pathology report confirmed the diagnosis of a malignant SFT.

NGS of the tissue of the SFT revealed a mutation in the BRCA1 (p.Q1458*) gene at the DNA level, and NAB2STAT6 fusion at the messenger RNA (mRNA) level (see Figure 4).

The patient's postoperative serum glucose levels 
A

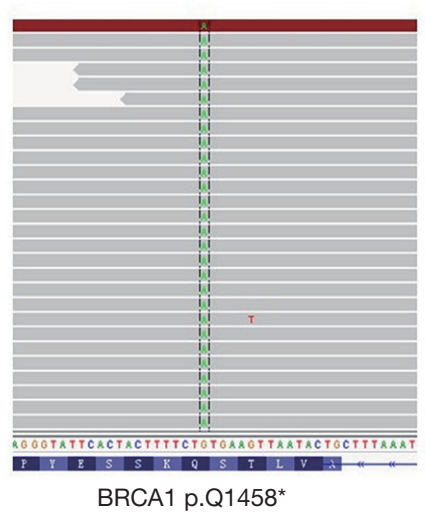

B

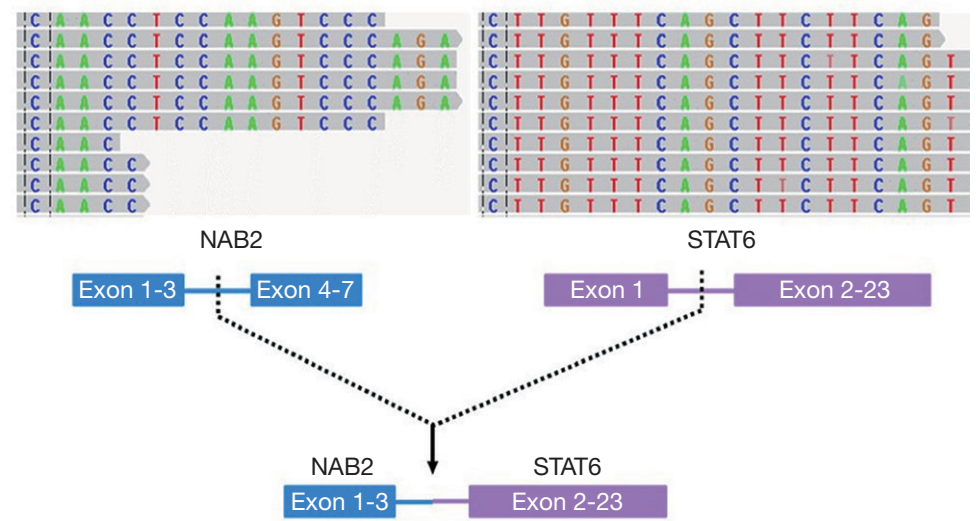

Figure 4 Next-generation sequencing (NGS) detected the breast cancer gene 1 (BRCA1) germline mutation and NAB2-STAT6 fusion transcripts of the intrathoracic solitary fibrous tumors (SFTs). NGS reads in the BRCA1 germline mutation and NAB2-STAT6 fusion breakpoint regions are visualized using the Integrated Genomics Viewer (Broad Institute, Cambridge, MA, United States).

normalized, and he experienced no hypoglycemic episodes. Additionally, the patient's serum IGF-II levels decreased from $1,228.71$ to $347.82 \mathrm{mg} / \mathrm{dL}$. After the operation, the case was submitted to a multidisciplinary team for discussion. The experts agreed with the approach adopted (i.e., that the tumor be removed completely), and that no additional adjuvant treatment was needed at present. The patient was followed up once every 4 months for the first 2 years and then, every 6 months for the next 3-5 years. The digital clubbing resolved within 3 months. No episodes of hypoglycemia occurred, and no recurrence or other signs of metastasis were found based on the CT examinations during the 1.5 year follow-up period.

All procedures performed in studies involving human participants were in accordance with the ethical standards of the institutional and/or national research committee(s) and with the Helsinki Declaration (as revised in 2013). Written informed consent was obtained from the patient.

\section{Discussion}

Pleural SFT is an uncommon primary tumor originating from the mesenchymal layer of the parietal or visceral pleura (3). The assessed age-standardized incidence ratio (World Standard Population) for pleural SFT is 2.8 per 100,00 cases (8). There is no gender predominance, and it peaks among patients aged $50-70$ years old, but has been reported in patients of all ages $(3,9,10)$. Most SFTs are benign (11). In relation to malignant SFTs, Penel et al. reported that they are hypercellular and display at least focal moderate-to-severe nuclear atypia. Malignant SFTs often have infiltrative margins with surrounding tissues, a high mitotic count ( $\geq 4$ mitoses per 10 high-power fields), and display cellular pleomorphism and tumor necrosis (11).

The clinical presentation of a SFT is of an asymptomatic slow growing mass that usually presents as an incidental finding on a radiological scan. When a tumor becomes large enough, the patient may present with local symptoms due to compression, such as vague chest pain, dyspnea, or a cough $(9,12)$. It has been reported that nearly a quarter of pleural SFTs are commonly associated with paraneoplastic syndromes $(4,9,13)$. Hypertrophic pulmonary osteoarthropathy (HPO), also referred to as PMBS, was first described by Bamberger in 1889, who described painful, digital clubbing; arthralgia; the swelling of the wrists, knees, ankle or elbows; and dermal hypertrophy (14-16). It has been reported that up to $22 \%$ of SFT patients have PMBS (9). The cause of HPO is not clearly understood. It has been posited that HPO may result from the following 3 pathophysiological causes: abnormal vascularization, hypoxia, and chronic inflammation, which all presumably give rise to a terminal common pathway involved in the vascular endothelial growth factor (16). In general, the HPO symptoms are effectively mitigated after the tumor is removed often within a few days but might reappear if the tumor recurs.

Reactive hypoglycemia, which was first described in 1930 by Doege and Potter independently, is connected with pleural SFTs in nearly $4 \%$ of cases $(5,9)$. Clinical manifestations of hypoglycemia consist of a number of 
symptoms, including diaphoresis, tremors, anxiety, and a loss of consciousness. Hypoglycemia is 2-3 times more common in women than men, and more common in rightsided pleural SFTs located in the right hemithorax (12). Previous reports have shown that large pleural SFTs $(>20 \mathrm{~cm})$ are often accompanied by hypoglycemia (4). The present case study involved a male patient suffering from a rightsided tumor. Hypoglycemia is thought to be the result of secretions of the precursor to insulin, such as factor-II (proIGF-II) (17). Pro-IGF-II can activate the insulin receptor, thus increasing peripheral glucose uptake and inhibiting hepatic gluconeogenesis, which results in hypoglycemia (17). A high IGF-II:IGF-I ratio is viewed as a surrogate marker of hypoglycemia. A ratio of 3:1 normally indicates hypoglycemia The IGF-II:IGF-I ratio is viewed as a surrogate marker with a high IGF-II concentration, in which 3:1 is the rate under normal consideration (18). In the present case, the patient's preoperative serum IGF-II level was $1,228.71 \mu \mathrm{g} / \mathrm{dL}$, but his serum IGF-I level was only $108 \mathrm{ng} / \mathrm{mL}$. Additionally, it is worth noting that high IGF-II may reduce the level of blood potassium in the short term (19). In this case, our patient presented with hypokalemia based on potassium concentrations of $2.67 \mathrm{mmol} / \mathrm{L}$. After surgery, his blood potassium level returned to normal. However, the mechanism underlying these findings remains unclear.

Imaging characteristics are important diagnostic tools. Both CT scanning and MRI are very effective at showing the size and location of tumors, the invasion of surrounding structures, and aid in surgical planning. CT scans of pleural SFTs typically show smooth, well-defined borders, and a homogenous mass with the same density as muscle. Mediastinum and lung parenchyma are often displaced under pressure $(2,9,20)$. A malignant SFT of the pleura usually has unclear boundaries, adhesion with or invasion of the surrounding tissues, uneven density, calcification, and pleural effusion (20). MRI can also be a useful alternative, as MRI usually shows multinodular tumors of low-signal intensity on T1-weighted images and high-signal intensity on T2-weighted images (7). Positron emission tomographyCT (PET-CT) usually shows low 18F-deoxyglucose uptake in the intrathoracic mass, but has limited diagnostic value for malignant pleural SFT patients $(21,22)$.

Pathological diagnosis is the gold standard for diagnosing pleural SFTs. The histopathological character of SFT indicates the existence of sparse and dense areas under the separation of fibrous stroma, and the mutual existence of hemangiopericytoma branching vessels (23). Standards of malignancy, as identified by England et al., include high cellularity and mitotic activity (over 4 mitotic figures for every 10 in a high-power field), pleomorphism, hemorrhage, and necrosis (12). De Perrot and other researchers have reported that malignant SFTs of the pleura have a high cellularity based on crowding and overlapping of nuclei, cellular pleomorphism, a high mitotic count, necrosis, and stromal/vascular invasion (19). In the present case, while some of the preoperative features of the tumor indicated a latent malignancy, the histologic analysis confirmed that the tumor was benign. Immunohistochemical analyses play an indispensable role in distinguishing pleural SFTs from other pleural tumors, such as mesothelioma, sarcoma, and other similar neoplasms. Pleural SFTs are positively correlated with vimentin, Bcl-2, CD34, and STAT6, but are negatively correlated with AE1/AE3, synaptophysin, S-100, and calretinin (7). In some cases, it has been suggested that in addition to CD34 and bcl-2, IGF-II should also be used as a marker for the postoperative differential diagnosis (24).

In addition, a gene analysis based on NGS might reveal the mutation features of pleural SFTs (25). Recently, an intrachromosomal gene fusion (a NAB2-STAT6 fusion) was identified as the defining driving genetic event of SFT, and different fusion types were correlated with tumor histology and behavior. Due to the proximity of NAB2 and STAT6 on chromosome 12, this fusion is difficult to identify by fluorescence in-situ hybridization (26). In a clinicopathologic study conducted by Tai et al., the NAB2STAT6 fusion was found in 34 of 52 cases (27). These studies have shown that NAB2-STAT6 gene fusion is the most prominent and well-adopted genomic hallmark of pleural SFTs. Additionally, given the nuclear entry of STAT6 driven by the NAB2-STAT6 gene fusion, several studies have reported the high sensitivity and specificity of STAT6 nuclear expression for SFTs (28-31). This is the first case to report germline BRCA1 functional mutation in pleural SFTs. BRCA1 is a tumor-suppressor gene that encodes a protein involved in DNA repair. Kang et al. suggested that the BRCA1 p.Q1458* mutation changes the glutamine residue in reference to the sequence to stopgained codon protein truncation (32). Abbas et al. suggested that the Q1458* mutation changes of BRCA1 are highly pathogenic in breast cancer patients (33). Genetic testing for BRCA1 p.Q1458* could be helpful in the diagnosis and treatment of cancer. However, in our case, the patient had no family history of cancer, including breast cancer.

A complete resection with wide margins of adjacent tissues is recommended for the treatment of pleural SFTs (12). Thoracotomy has been employed as therapy for 
those who suffer from a large tumor or expansive adhesions. Following the complete resection of the tumor, the deformed lung tissue may expand, and the paraneoplastic syndromes usually disappear (32). To date, findings on the effects of adjunctive therapy, including chemotherapy, radiation, and embolization, have been controversial (7).

The surgical removal of pleural SFTs usually produces satisfactory results, including a 5-year disease-free survival rate of approximately $80 \%$. However, the recurrence rate of malignant SFTs of the pleura has been reported to be $63 \%$, even after complete resection (34). Tapias et al. developed a scoring system to predict the recurrence of SFTs of the pleura after resection, including parietal pleural origin (vs. visceral/intrapulmonary origin), sessile morphology (vs. pedunculated morphology), a size larger than $10 \mathrm{~cm}$ ( $v s$. a size smaller than $10 \mathrm{~cm})$, hypercellularity, the presence of necrosis or hemorrhage, and the number of mitoses (per 10 in a high-power field) (35). This system partially overlaps with the diagnosis criteria of malignancy, but it emphasizes predictive value. A long-term follow-up period is mandatory due to the risk of recurrence of SFTs. The survival outcomes revealed that the 5-year recurrencefree survival and overall survival (OS) rates of benign patients were both $100 \%$; however, the 5-year relapsefree survival (RFS) and OS rates of malignant patients were $58.3 \%$ and $66.7 \%$, respectively (3). Malignant SFT recurrence has been reported 17 years after surgical resection $(3,36)$. Thus, a postoperative follow-up period with CT is recommended every 3-6 months for 2 years and annually thereafter. For malignant tumors with a high risk of recurrence, a closer clinical follow-up approach is recommended.

\section{Acknowledgments}

We would like to thank Geneseeq for performing nextgeneration sequencing for our patient.

Funding: This work was supported by the National Key R\&D Program (2018YFC1312105), the Institutional Fundamental Research Funds (20182018PT32033), the Ministry of Education Innovation Team Development Project (IRT-17R10), the Beijing Hope Run Special Fund of Cancer Foundation of China (LC2019B15).

\section{Footnote}

Reporting Checklist: The authors have completed the CARE reporting checklist. Available at https://dx.doi. org/10.21037/atm-21-914

Peer Review File: Available at https://dx.doi.org/10.21037/ atm-21-914

Conflicts of Interest: All authors have completed the ICMJE uniform disclosure form (available at https://dx.doi. org/10.21037/atm-21-914). The authors have no conflicts of interest to declare.

Ethical Statement: The authors are accountable for all aspects of the work in ensuring that questions related to the accuracy or integrity of any part of the work are appropriately investigated and resolved. All procedures performed in studies involving human participants were in accordance with the ethical standards of the institutional and/or national research committee(s) and with the Helsinki Declaration (as revised in 2013). Written informed consent was obtained from the patient.

Open Access Statement: This is an Open Access article distributed in accordance with the Creative Commons Attribution-NonCommercial-NoDerivs 4.0 International License (CC BY-NC-ND 4.0), which permits the noncommercial replication and distribution of the article with the strict proviso that no changes or edits are made and the original work is properly cited (including links to both the formal publication through the relevant DOI and the license). See: https://creativecommons.org/licenses/by-nc-nd/4.0/.

\section{References}

1. Davanzo B, Emerson RE, Lisy M, et al. Solitary fibrous tumor. Transl Gastroenterol Hepatol 2018;3:94.

2. Sergew A, Merrick D, Chan ED. A 64-year-old man with joint swelling and an abnormal chest radiograph. Chest 2008;133:805-8.

3. Zhou C, Li W, Shao J, et al. Thoracic solitary fibrous tumors: an analysis of 70 patients who underwent surgical resection in a single institution. J Cancer Res Clin Oncol 2020;146:1245-52.

4. Balduyck B, Lauwers P, Govaert K, et al. Solitary fibrous tumor of the pleura with associated hypoglycemia: Doege-Potter syndrome: a case report. J Thorac Oncol 2006;1:588-90.

5. Doege KW. Fibro-sarcoma of the mediastinum. Ann Surg 1930;92:955.

6. Meng $\mathrm{W}$, Zhu HH, Li H, et al. Solitary fibrous tumors 
of the pleura with Doege-Potter syndrome: a case report and three-decade review of the literature. BMC Res Notes 2014;7:515.

7. Han G, Zhang Z, Shen X, et al. Doege-Potter syndrome: a review of the literature including a new case report. Medicine (Baltimore) 2017;96:e7417.

8. Thorgeirsson T, Isaksson HJ, Hardardottir H, et al. Solitary fibrous tumors of the pleura: an estimation of population incidence. Chest 2010;137:1005-6.

9. Sun ZG, Wang Z, Zhang M. A 70-year-old man with hypoglycemia, clubbing of fingers and toes, and a large mass of the right hemithorax. Chest 2011;139:1528-31.

10. Lococo F, Cesario A, Cardillo G, et al. Malignant solitary fibrous tumors of the pleura: retrospective review of a multicenter series. J Thorac Oncol 2012;7:1698-706.

11. Penel N, Amela EY, Decanter G, et al. Solitary fibrous tumors and so-called hemangiopericytoma. Sarcoma 2012;2012:690251.

12. England DM, Hochholzer L, McCarthy MJ. Localized benign and malignant fibrous tumors of the pleura: a clinicopathologic review of 223 cases. Am J Surg Pathol 1989;13:640-58.

13. Dong SS, Wang N, Yang CP, et al. Giant Cell-Rich Solitary Fibrous Tumor in the Nasopharynx: Case Report and Literature Review. Onco Targets Ther 2020;13:6819-26.

14. Bamberger E. Sitzung der KK Ges. der Aerzte in Wien, 8 März 1889. Wien Klin Wschr 1889;2:226.

15. Myers KA, Farquhar DR. Does this patient have clubbing? JAMA 2001;286:341-7.

16. Callemeyn J, Van Haecke P, Peetermans WE, et al. Clubbing and hypertrophic osteoarthropathy: insights in diagnosis, pathophysiology, and clinical significance. Acta Clinica Belgica 2016;71:123-30.

17. Tsuro K, Kojima H, Okamoto S, et al. Glucocorticoid therapy ameliorated hypoglycemia in insulin-like growth factor-II-producing solitary fibrous tumor. Intern Med 2006;45:525-9.

18. Teale JD, Marks V. Inappropriately elevated plasma insulin-like growth factor II in relation to suppressed insulin-like growth factor I in the diagnosis of non-islet cell tumour hypoglycaemia. Clin Endocrinol (Oxf) 1990;33:8798.

19. de Perrot M, Fischer S, Bründler MA, et al. Solitary fibrous tumors of the pleura. Ann Thorac Surg 2002;74:285-93.

20. Gupta A, Souza C, Sekhon H, et al. Solitary fibrous tumour of pleura: CT differentiation of benign and malignant types. Clin Radiol 2017;72:796.e9-796.e17.

21. Kitada M, Yasuda S, Takahashi N, et al. Non-islet cell tumor hypoglycemia caused by intrathoracic solitary fibrous tumor: a case report. J Cardiothorac Surg 2016;11:49.

22. Tazeler Z, Tan G, Aslan A, et al. The utility of 18F-FDG PET/CT in solitary fibrous tumors of the pleura. Rev Esp Med Nucl Imagen Mol 2016;35:165-70.

23. Huang SC, Huang HY. Solitary fibrous tumor: An evolving and unifying entity with unsettled issues. Histol Histopathol 2019;34:313-34.

24. Corti B, Carella R, Gabusi E, et al. Solitary Fibrous Tumour of the Urinary Bladder with Expression of bcl-2, CD34, and Insulin-Like Growth Factor Type II. Eur Urol 2001;39:484-8.

25. Song Z, Yang F, Zhang Y, et al. Surgical therapy and nextgeneration sequencing-based genetic alteration analysis of malignant solitary fibrous tumor of the pleura. Onco Targets Ther 2018;11:5227.

26. Guseva NV, Tanas MR, Stence AA, et al. The NAB2STAT6 gene fusion in solitary fibrous tumor can be reliably detected by anchored multiplexed PCR for targeted next-generation sequencing. Cancer Genet 2016;209:303-12.

27. Tai HC, Chuang IC, Chen TC, et al. NAB2-STAT6 fusion types account for clinicopathological variations in solitary fibrous tumors. Mod Pathol 2015;28:1324-35.

28. Schweizer L, Koelsche C, Sahm F, et al. Meningeal hemangiopericytoma and solitary fibrous tumors carry the NAB2-STAT6 fusion and can be diagnosed by nuclear expression of STAT6 protein. Acta Neuropathol 2013;125:651-8.

29. Huang SC, Li CF, Kao YC, et al. The clinicopathological significance of NAB 2-STAT 6 gene fusions in 52 cases of intrathoracic solitary fibrous tumors. Cancer Med 2016;5:159-68.

30. Doyle LA, Vivero M, Fletcher CD, et al. Nuclear expression of STAT6 distinguishes solitary fibrous tumor from histologic mimics. Mod Pathol 2014;27:390-5.

31. Yoshida A, Tsuta K, Ohno M, et al. STAT6 immunohistochemistry is helpful in the diagnosis of solitary fibrous tumors. Am J Surg Pathol 2014;38:552-9.

32. Kang PCE, Phuah SY, Sivanandan K, et al. Recurrent mutation testing of BRCA1 and BRCA2 in Asian breast cancer patients identify carriers in those with presumed low risk by family history. Breast Cancer Res Treat 2014;144:635-42.

33. Abbas S, Siddique A, Shahid N, et al. Breast cancer 
risk associated with BRCA1/2 variants in the Pakistani population. Breast Cancer 2019;26:365-72.

34. Fukai R, Irie Y, Watanabe H. Surgically cured paraneoplastic hypoglycemia associated with solitary fibrous tumor of the pleura: report of two cases. Clin Case Rep 2017;5:1119.

35. Tapias LF, Mercier O, Ghigna MR, et al. Validation of a scoring system to predict recurrence of resected solitary fibrous tumors of the pleura. Chest 2015;147:216-23.

36. Kovacs T, Waxman J. Recurrence of a malignant solitary fibrous tumor of the pleura 17 years after primary tumor resection - A case report. Respir Med Case Rep 2019;28:100895.

(English Language Editors: L. Huleatt and J. Gray)

Cite this article as: Guo W, Ji Y, Guo L, Che S, Huai Q, Yang K, Tan F, Xue Q, Gao S, He J. Severe hypoglycemia and finger clubbing in a patient with a BRCA1 mutation in a solitary fibrous tumor: a case report. Ann Transl Med 2021;9(13):1093. doi: 10.21037/atm-21-914 